\title{
SCREENING
}

\section{Improved model for lung cancer detection}

The National Lung Cancer Screening Trial (NLST) previously showed that lowdose CT screening for lung cancer can reduce mortality by $20 \%$. Consequently, some organizations have recommended that this approach is adopted for individuals at high risk of lung cancer. The NLST criteria for screening include age (55-74 years), history of smoking of at least 30 pack-years, and 15 years or fewer since smoking cessation. Although these criteria are intended to improve the number of cancers detected, they exclude other risk factors, such as BMI and family history of lung cancer. Thus, applying a more-accurate risk-prediction model for population screening would help identify those at highest risk.

In 2011, Martin Tammemägi and coauthors developed and validated a lung cancer risk-prediction model using data collected from the Prostate, Lung, Colorectal and Ovarian (PLCO) screening trial. Now, Tammemägi and his team have modified and updated their model to make it directly applicable to the NLST data. Tammemägi describes the rationale behind the latest study, "we wanted to improve this model and make it more practical, and apply it to lung cancer screening to help make screening more efficient."

In this study, data were available for 51,033 NLST participants and 73,618 smokers in the PLCO study. The researchers used a logistic regression approach, and to describe nonlinear relationships they "used multivariable fractional polynomials, which work very nicely without overfitting models, as some other methods are prone to." Tammemägi highlights the main study findings: "This is the first time it has been demonstrated that using an accurate risk prediction model is significantly superior in selecting candidates for lung cancer screening over the current standard method employing the NLST criteria."

A previous study showed that 12,000 lung cancer deaths could be averted each year in the USA by applying CT screening to everyone who qualified according to the NLST criteria. By extrapolating the numbers of patients from this other study and applying the PLCO model to detect those at highest risk, an estimated 2,764 additional lives could be saved. "If CT lung cancer screening becomes a widespread public health practice, selection of individuals for

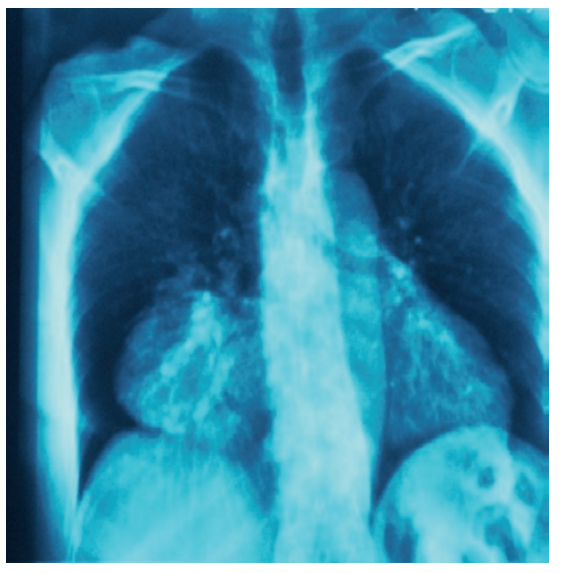

screening using an accurate lung cancer risk prediction model is expected to save additional lives and make screening more cost effective," notes Tammemägi. His team and other collaborators are already working on publishing risk models based on prospective population-based screening data to help provide some guidance to clinicians as a result of the large number of false-positive screens.

Lisa Hutchinson

Original article Tammemägi, M. C. et al. Selection criteria for lung-cancer screening. N. Engl. J. Med. 368, 728-736 (2013) 